\title{
BOÖTES II REBOÖTED: AN MMT/MEGACAM STUDY OF AN ULTRAFAINT MILKY WAY SATELLITE
}

\author{
S. M. Walsh, ${ }^{1}$ B. Willman, ${ }^{2,3}$ D. SAnd ${ }^{4,5}$ J. Harris, ${ }^{4}$ A. Seth, ${ }^{2}$ D. Zaritsky, ${ }^{4}$ And H. JerJen ${ }^{1}$ \\ Received 2007 December 18; accepted 2008 July 21
}

\begin{abstract}
We present MMT/Megacam imaging in Sloan $g$ and $r$ of the extremely low luminosity Boötes II Milky Way companion. We use a bootstrap approach to perform robust measurements of, and to calculate uncertainties on, Boötes II's distance, luminosity, size, and morphology. Comparisons with theoretical isochrones and empirical globular cluster fiducials show that Boötes II's stellar population is old and metal-poor $([\mathrm{Fe} / \mathrm{H}] \lesssim-2)$. Assuming a stellar population like that of M92, Boötes II is at a distance of $42 \pm 2 \mathrm{kpc}$, closer than the initial published estimate of $60 \pm 10 \mathrm{kpc}$. This distance revision, combined with a more robust measurement of Boötes II's structure with both a Plummer model and an exponential model, results in more compact inferred physical half-light sizes of $r_{h} \simeq 36 \pm 9$ and $33 \pm 10 \mathrm{pc}$, respectively, and lower-limit inferred luminosities of $M_{V} \simeq-2.4 \pm 0.7$ and $-2.2 \pm 0.7 \mathrm{mag}$, respectively. Our revised size and luminosity calculations move Boötes II into a region of size-luminosity space not previously known to be occupied by old stellar populations, but also occupied by the recently discovered Milky Way satellites Willman 1 and Segue 1. We show that the apparently distorted morphology of Boötes II is not statistically significant given the present data. We use a tidal argument to support a scenario in which Boötes II is a dwarf galaxy (dark matterdominated) rather than a globular cluster (not dark matter-dominated), although our inferred uncertainty on the $M / L$ for Boötes II is substantial. Moreover, we cannot rule out the possibility that Boötes II is a star cluster on the verge of disruption like Palomar 5.
\end{abstract}

Subject headings: galaxies: dwarf — Local Group

Online material: color figures

\section{INTRODUCTION}

Over the last five years, the Sloan Digital Sky Survey (SDSS) has been extensively searched for extremely low surface brightness dwarf spheroidal galaxies. These searches use the catalog of stellar sources to identify spatial overdensities of the old, metalpoor stars characteristic of these dwarfs. To date, these searches (e.g. Willman et al. 2002; Koposov et al. 2007a) have resulted in the discoveries of 14 new Milky Way satellites.

Most of these new objects have total luminosities less than the median luminosity of the Milky Way's globular clusters $\left(M_{V} \sim\right.$ -7), but have sizes characteristic of known dwarf spheroidals $\left(r_{\text {half }} \gtrsim 100 \mathrm{pc}\right)$, complicating their classifications as either star clusters or dwarf galaxies. In this paper, we assert that the physical distinction between a globular cluster and a dwarf galaxy is that a dwarf galaxy is, or was at some point, the primary baryonic component of a dark matter halo, whereas a globular cluster was not. Nine of the 14 new satellites (Boötes, Canes Venatici, Canes Venatici II, Coma Berenices, Hercules, Leo IV, Leo T, Ursa Major, and Ursa Major II) were originally classified as dwarf spheroidals because they had scale sizes $\gtrsim 100$ pc (Belokurov et al. 2006, 2007; Zucker et al. 2006a, 2006b; Sakamoto \& Hasegawa 2006; Willman et al. 2005b). Follow-up spectroscopic studies demonstrated that they indeed appear to require dark matter to explain their kinematics, securing their classification as dwarf galaxies (Simon \& Geha 2007; Martin et al. 2007; Strigari et al. 2007a; Walker et al. 2007). Koposov 1 and 2 (Koposov et al. 2007b)

\footnotetext{
1 Research School of Astronomy and Astrophysics, Australian National University, Cotter Road, Weston, ACT 2611; swalsh@mso.anu.edu.au.

2 Harvard-Smithsonian Center for Astrophysics, 60 Garden Street Cambridge, MA 02138.

3 Clay Fellow; beth.willman@gmail.com.

4 Steward Observatory, University of Arizona, 933 North Cherry Avenue, Tucson, AZ 85721.

5 Chandra Fellow.
}

have scale sizes of only $3 \mathrm{pc}$, and have thus been classified as new globular clusters.

The classification of the other three new satellites, Segue 1, Willman 1, and Boötes II $\left(M_{V} \sim-2.5\right.$; Belokurov et al. 2007; Willman et al. 2005a; Walsh et al. 2007), has been less straightforward. They are old stellar populations with intermediate sizes between known Milky Way globular clusters and dwarf spheroidals, but they have fewer stars than nearly any known galaxy or globular cluster. Although initial estimates based purely on SDSS data placed Boötes II close to Coma Berenices in size-luminosity space at $\log \left(r_{h} / 1 \mathrm{pc}\right)=1.85, M_{V}=-3.1$, in this paper we present estimates based on a more robust algorithm and on deeper, MMT/MegaCam imaging in $g$ and $r$, which shift Boötes II's size and luminosity closer to that of Willman 1 and Segue 1.

Despite its tiny luminosity, spectroscopic $[\mathrm{Fe} / \mathrm{H}]$ estimates and kinematic studies of Willman 1 have supported the idea that it may require dark matter to explain its properties, thus classifying it as a dwarf galaxy (Martin et al. 2007; Strigari et al. 2008). Whether or not these three Milky Way companions with only $\sim 1000 L_{\odot}$ are galaxies or globular clusters is of fundamental import to both our understanding of galaxy formation at the smallest scales and to our understanding of the size and mass scale of dark matter clustering. Although the extent to which tides have affected Willman 1's present-day luminosity is uncertain, this object raises some questions. Are we seeing for the first time the low-luminosity and mass limits of galaxy formation? If so, what properties can we infer for the dark matter halos that host such galaxies? Segue I and Boötes II presently lack their own published spectroscopic studies with which to evaluate these scenarios. However, rigorous derivations of the detection limits of the most recent SDSS searches for such objects (Koposov et al. 2007a; Walsh et al. 2008) show that many similar objects could remain yet undiscovered around the Galaxy, underscoring the importance of understanding their physical properties. 
With the study presented in this paper, we aim to provide the first robust measurements of the basic properties (distance, luminosity, and structure) of Boötes II and its stellar population, and to evaluate the present evidence for its classification. In $\S 2$, we describe MMT/ MegaCam observations of Boötes II, data reduction, and artificial star tests. In $\S 3$, we use these data to derive revised estimates of Boötes II's properties, to verify that its stellar population is old and metal-poor, and to investigate whether it has a distorted morphology. We discuss these results and evaluate evidence for a dwarf galaxy versus globular cluster classification of Boötes II in $\S 4$.

\section{DATA}

We observed Boötes II on 2007 June 5 with MegaCam (McLeod et al. 2000) on the MMT. These data were obtained as part of a larger survey program to image ultrafaint Milky Way satellites with MMT/Megacam. MMT/MegaCam has 36 chips with $2048 \times 4608$ pixels of $0.08^{\prime \prime}$ pixel $^{-1}$, for a total field of view (FOV) of $24^{\prime}$. We obtained five $180 \mathrm{~s}$ dithered exposures in Sloan $g$, and five $240 \mathrm{~s}$ dithered exposures in Sloan $r$ in gray conditions, with $1.0^{\prime \prime}-1.2^{\prime \prime}$ image quality in the $g$ images and $0.9^{\prime \prime}-1.0^{\prime \prime}$ image quality in the $r$ images. We reduced the data based on the method described in Matt Ashby's Megacam Reduction Guide. ${ }^{6}$ Our reduction relied in part on software written specifically for MMT/ MegaCam data reduction by Brian McLeod. We used the SDSS Data Release 6 (DR6; York et al. 2000; Adelman-McCarthy et al. 2008) stellar catalog to derive precise astrometric solutions for each science exposure. We also used the SDSS catalog to derive an illumination correction in $g$ and $r$ that divides out the variation in zero point across MegaCam's FOV. We used local copies of the SDSS data set, maintained at the Harvard-Smithsonian Center for Astrophysics.

We did a weighted coaddition of the reduced images using SWARP, ${ }^{7}$ and then used the DAOPHOT II/Allstar package (Stetson 1994) to do point-source photometry on the resulting images. We visually verified the integrity of the shape and full width at halfmaximum (FWHM) of the point spread functions (PSFs) in the stacked images across the $24^{\prime}$ FOV. Photometry was carried out using a method similar to that of Harris (2007), except that we used the command line versions of DAOPHOT and Allstar rather than the IRAF versions.

To derive the photometric calibration for our data, we first matched the SDSS stellar catalog to the Allstar catalog for these new observations. We used the 91 SDSS stars within our FOV with $18<r<21$ and $0.1<g-r<0.8$ to perform the photometric calibration. We limited the calibration to stars fainter than $r=18$ mag to avoid the saturation limit of the MegaCam data. We limited the calibration to stars with colors between $0.1<g-$ $r<0.8$, because the Boötes II member stars resolved in this study (with the exception of a few possible blue horizontal branch stars) all have $0.1<g-r<0.6$. There were insufficient SDSS stars in our FOV bluer than 0.2 mag to determine whether our derived calibration is appropriate for very blue stars.

We then did a linear least-squares fit for the zero points and color terms, including uncertainties in color and magnitudes on each star, and throwing out $3 \sigma$ outliers:

$$
\begin{aligned}
& g=g_{\text {instr }}+7.27( \pm 0.029)+0.091( \pm 0.068)(g-r), \\
& r=r_{\text {instr }}+7.33( \pm 0.025)+0.074( \pm 0.054)(g-r) .
\end{aligned}
$$

Uncertainties were derived from a 1000 iteration bootstrap of the data. In addition, there is uncertainty in the SDSS zero points themselves of about $0.01 \mathrm{mag}$ (Padmanabhan et al. 2008).

\footnotetext{
${ }_{7}^{6}$ See http://www.cfa.harvard.edu/ mashby/megacam/megacam_frames.html. See http://terapix.iap.fr/soft/swarp.
}

Throughout this paper, we adopt SDSS photometry rather than MegaCam photometry for stars brighter than $r=18.0$ mag. All magnitudes in this paper have been extinction corrected with values from the Schlegel et al. (1998) dust maps provided in the SDSS catalog; the median $E(g-r)$ along the line of sight to Boötes II is $0.02 \mathrm{mag}$.

We use artificial star tests to measure the photometric errors and completeness as a function of position in the $g-r$ colormagnitude diagram (CMD). Artificial stars are constructed from the $g$ and $r$ PSFs measured during the data reduction process, and injected into the coadded $g$ and $r$ images using a uniform grid with spacing in $x$ and $y$ equal to 10 times the FWHM of the PSF (so that artificial stars overlap only beyond their $10 \sigma$ radii). This fixed geometry imposes a limit of about 18,500 on the number of artificial stars that can be added to the image. To build up our number statistics, we inject artificial stars into 20 copies of the $g$ and $r$ images, randomly offsetting the grid's zero-point position in $x$ and $y$ for each iteration. This results in a total sample of 370,000 artificial stars. The $r$ photometry of the artificial stars is drawn randomly from $\sim 18$ to $28 \mathrm{mag}$, with an exponentially increasing probability toward fainter magnitudes. To properly characterize the tail of the completeness function and the impact of blends on the photometric errors of faint objects, we simulate stars up to 3 mag fainter than the nominal faint limit. The $g-r$ color is then drawn randomly over the range -0.5 to $1.5 \mathrm{mag}$ to determine the $g$-magnitude. We photometer the artificial star images with the same photometry pipeline as we used on the science frames.

If an input artificial star is not present in either the $g$ or $r$ Allstar files, then it is flagged as a nondetection for calculation of the completeness rate. We applied a strict cut of the DAOPHOT sharpness parameter between -1 and 1 to determine whether a star would be included in our analysis; this was done both for the actual Boo II data and for the artificial star tests. Although this strict sharpness cut yields completeness limits that are brighter than if we use no cut at all, we found it necessary to eliminate many galaxy interlopers and to provide an improved measurement of Boötes II's stellar population without sacrificing much precision in our quantitative results. At a $g-r$ color of $0.25 \mathrm{mag}$, the $50 \%$ and $90 \%$ completeness limits of these data are $r=23.5$ and 22.9 mag, respectively.

Figure 1 shows the CMDs of stars within $4.4^{\prime}$ of the center of Boötes II from the SDSS DR6 data and from the MMT/Megacam data. The $50 \%$ completeness, as a function of color, is overplotted on the Megacam CMD.

\section{BOÖTES II PROPERTIES}

\subsection{Bootstrap Analysis}

With only $\sim 100$ object stars resolved in this study, smallnumber statistics will constitute a substantial, if not dominant, source of uncertainty in the derived quantities. For the ambiguous ultrafaint satellites such as Boötes II, a rigorous examination of the uncertainties is essential to measure any of their properties. We use a 10,000 iteration bootstrap analysis to determine both the most likely values of Boötes II's properties and the uncertainties associated with each measurement, as detailed in the following sections. For each iteration, the data are randomly resampled with replacement and then (as described in the remainder of $\S 3$ ) analyzed in order to derive the central right ascension and declination, the distance modulus, the Plummer and exponential half-light radii and total absolute magnitudes, the King core and tidal radius, the position angle, the ellipticity, and the asymmetry. Aside from the King tidal radius, the bootstrapped distributions of the derived parameters are well described by a 

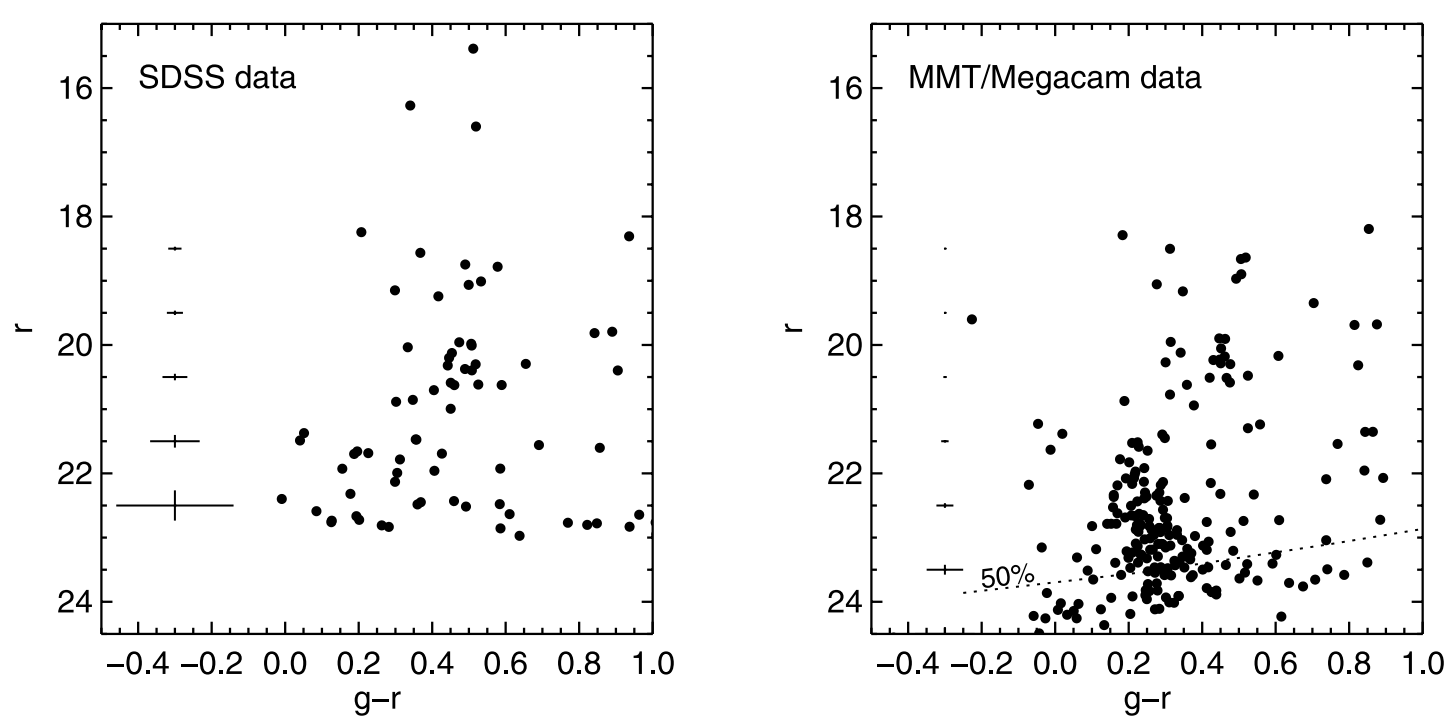

FIG. 1.-Color-Magnitude Diagrams (CMDs) of stars within 4.5' ( 1.5 Plummer half-light radii) of the center of Boötes II, based on MMT/Megacam observations. Left: Data from the SDSS DR6. Right: MMT/Megacam data. Error bars showing the color and magnitude uncertainties as a function of $r$ are overplotted.

Gaussian. All quoted values and uncertainties are thus the peaks and standard deviations of the bootstrap distributions. For the King tidal radius, we quote the half-width at half-maximum as the uncertainty, because there is a long, poorly populated tail of values that extends to high tidal radius. We report the values of the bootstrapped sample fits in Table 1.

\subsection{Central Position}

Boötes II contains no detectable unresolved luminous component, so we determine its center by locating the barycenter of likely member stars within a $4.5^{\prime}$ radius around the center published in Walsh et al. (2007). We define likely member stars as those that have colors and magnitudes consistent with M92 at an initial estimated distance modulus. We repeat this procedure with the returned right ascension and declination until the difference between input and output values converges. Our derived values are presented in Table 1 along with their associated bootstrap uncertainty. The uncertainties on the center of Boötes II are sub- stantial: $7.2 \mathrm{~s}$ in right ascension and $23^{\prime \prime}$ in declination. Because all parameters are derived for each of the bootstrapped samples, these uncertainties on the center are automatically propagated through to the uncertainties in Boötes II's structural parameters.

\subsection{Distance}

To investigate the distance to Boötes II's stellar population, we first compare its CMD to the empirical globular cluster fiducials M92, M3, M13, and M71 with $-2.4<[\mathrm{Fe} / \mathrm{H}]<-0.7$. We use $m-M=14.60,15.14,14.42$, and 13.71, respectively, for the four clusters (Paust et al. 2007; Kraft \& Ivans 2003; Cho et al. 2005; Grundahl et al. 2002). We choose to rely on fiducials, rather than theoretical isochrones, because these well-studied globular clusters have photometry in the exact photometric system to which we have calibrated our data. The fiducials we use are based on those of Clem et al. (2008) in Sloan $g^{\prime}-r^{\prime}$. They were converted into $g-r$ using the transformation of Rider et al. (2004) and checked by comparing the transformed fiducials directly to

TABLE 1

Boötes II Properties

\begin{tabular}{|c|c|c|c|}
\hline Parameter & Measured & Uncertainty & Bootstrap Median \\
\hline R.A. & $13^{\mathrm{h}} 58^{\mathrm{m}} 05.1^{\mathrm{s}}$ & $\pm 7.2 \mathrm{~s}$ & $13^{\mathrm{h}} 58^{\mathrm{m}} 04.3^{\mathrm{s}}$ \\
\hline 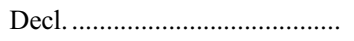 & $+12^{\circ} 51^{\prime} 31^{\prime \prime}$ & $\pm 23^{\prime \prime}$ & $+12^{\circ} 51^{\prime} 09^{\prime \prime}$ \\
\hline$(l, b)$ & $(353.75,68.86)$ & .. & ... \\
\hline$(m-M)$ & 18.1 & \pm 0.06 & 18.1 \\
\hline 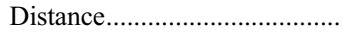 & $42 \mathrm{kpc}$ & $\pm 1.6 \mathrm{kpc}$ & $42 \mathrm{kpc}$ \\
\hline \multirow[t]{2}{*}{ 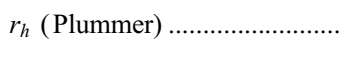 } & $2.8^{\prime}$ & \pm 0.7 & $2.8^{\prime}$ \\
\hline & $35 \mathrm{pc}$ & \pm 9 & $36 \mathrm{pc}$ \\
\hline \multirow[t]{2}{*}{ 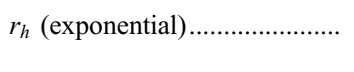 } & $2.5^{\prime}$ & \pm 0.8 & $2.6^{\prime}$ \\
\hline & $31 \mathrm{pc}$ & \pm 10 & $33 \mathrm{pc}$ \\
\hline$M_{V}$ (Plummer) $\ldots \ldots \ldots \ldots \ldots \ldots$ & -2.3 & \pm 0.7 & -2.4 \\
\hline 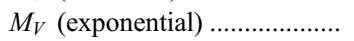 & -2.2 & \pm 0.7 & -2.2 \\
\hline 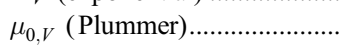 & $27.76 \mathrm{mag} \operatorname{arcsec}^{-2}$ & \pm 0.31 & $27.93 \mathrm{mag} \mathrm{arcsec}^{-2}$ \\
\hline$\mu_{0, V}($ exponential) $\ldots \ldots \ldots \ldots \ldots \ldots \ldots$ & $27.70 \mathrm{mag} \operatorname{arcsec}^{-2}$ & \pm 0.33 & $27.90 \mathrm{mag} \operatorname{arcsec}^{-2}$ \\
\hline$r_{c}$ & $25 \mathrm{pc}$ & \pm 9 & $25 \mathrm{pc}$ \\
\hline 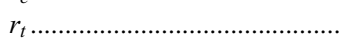 & $127 \mathrm{pc}$ & \pm 35 & $155 \mathrm{pc}$ \\
\hline$\theta$ & $-33^{\circ}$ & $\pm 57^{\circ}$ & $-28.5^{\circ}$ \\
\hline 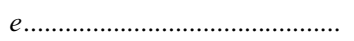 & 0.27 & \pm 0.15 & 0.34 \\
\hline$A_{\text {major }}$ & $\ldots$ & \pm 1.01 & -0.26 \\
\hline$A_{\operatorname{minor}}$ & $\ldots$ & \pm 1.03 & -0.33 \\
\hline
\end{tabular}




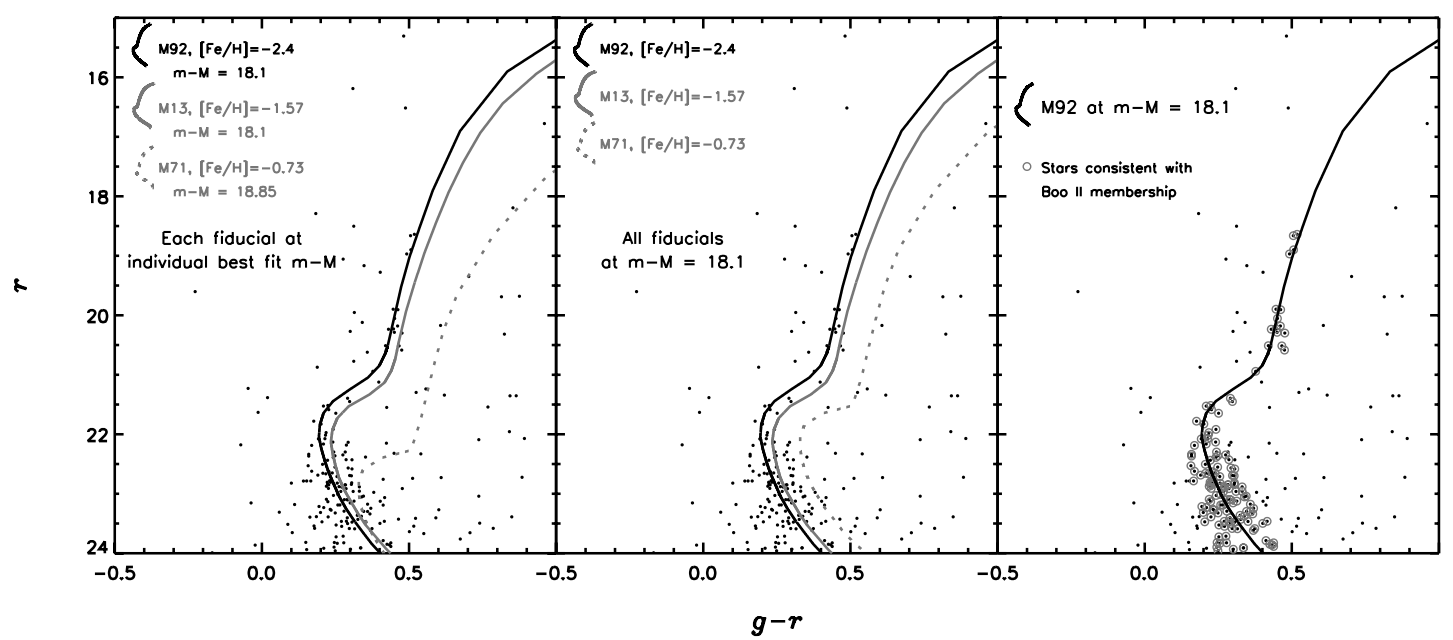

Fig. 2.-Color-Magnitude Diagrams of stars within $4.5^{\prime}$ of the center of Boötes II. Left: Globular cluster (GC) fiducials overplotted at their own best-fit distance modulus. Middle: GC fiducials overplotted at the M92 best-fit distance modulus of $m-M=18.1$. Right: M92 fiducial at $m-M=18.1$, with probable Boötes II member stars highlighted. [See the electronic edition of the Journal for a color version of this figure.]

the SDSS imaging of the clusters in the SDSS DR6 (J. Strader 2007, private communication). The robustness of our comparison depends on Boötes II having an old stellar population like those in these four comparison clusters. We address and confirm this with theoretical isochrones in $\S 3.4$.

For each fiducial, we find the distance modulus that provides the best fit to the stars in the CMD shown in the right panel of Figure 1. This CMD includes all stars within $4.5^{\prime}$ of Boötes II. To determine this distance modulus, we step each fiducial through $0.05 \mathrm{mag}$ intervals in $(m-M)$ from 17.5 to $19.5 \mathrm{mag}$, and find the number of stars brighter than $r=23.5$ that, considering color uncertainties, have colors within 0.05 mag of the fiducial. To eliminate the contribution of stars belonging to the thick disk and halo, we then do the same for stars exterior to $9.0^{\prime}$ and subtract this value normalized to an area of $\pi\left(4.5^{\prime}\right)^{2}$. We take the best fit for each fiducial as the distance modulus that maximizes this number of stars. The best-fit distance moduli for the M92, M3, M13, and M71 fiducials are 18.1,18.1, 18.1, and 18.85, with 96, 77, 87 , and 43 stars, respectively.

The left panel of Figure 2 shows the M92, M13, and M71 fiducials overplotted on the MMT CMD at their individual bestfit distances. For simplicity, we include M13 but not M3, because they have similar $[\mathrm{Fe} / \mathrm{H}]$, and because M13 provides a better match both quantitatively and qualitatively. In the central panel of Figure 2, we overplot the empirical M92, M13, and M71 fiducials, all projected to the M92 best-fit distance modulus of $m-$ $M=18.1(42 \mathrm{kpc})$. These confirm that it is reasonable to infer that the stellar population of Boötes II is like that of M92. We therefore use the M92 fiducial in our bootstrap analysis to derive the best-fit distance, which yields a distance modulus of $m-M=18.1 \pm 0.06$, which only includes the formal bootstrap uncertainty stemming from small-number statistics. We add in quadrature the distance modulus uncertainty of M92 (0.09 mag; Paust et al. 2007) and the $r$ zero-point uncertainty $(0.025 \mathrm{mag}$; $\S 2$ ) to derive $m-M=18.1 \pm 0.06$, or $d=42 \pm 2 \mathrm{kpc}$. If Boötes II had a stellar population different from M92, then the uncertainty in the distance would be larger.

\subsection{Stellar Population}

M92 has a very low $[\mathrm{Fe} / \mathrm{H}]$ of -2.4 and is $\alpha$-element enhanced relative to solar $([\mathrm{Ca} / \mathrm{Fe}]=0.3$; Sneden et al. 2000). The match between M92 and Boötes II's stellar populations thus sup- ports $[\mathrm{Fe} / \mathrm{H}]_{\text {BoolI }} \lesssim-2$, even if Boötes II is $\alpha$-depleted relative to M92 (which is typical of the contrast between dwarf spheroidal galaxies and globular cluster populations; Pritzl et al. 2005). Figure 2 shows that M13's fiducial sequence is only $0.04 \mathrm{mag}$ redder in $(g-r)$ than that of M92 below the main-sequence turnoff. The uncertainties in the $g$ and $r$ zero points of the MegaCam data in Figure 2 result in a $(g-r)$ calibration that is uncertain at $0.038 \mathrm{mag}$. These Boötes II data are thus also consistent with a more moderate abundance $([\mathrm{Fe} / \mathrm{H}] \sim-1.6)$. However, using independent $V$ and $I$ observations obtained on VLT FORS2, H. Jerjen et al. (in preparation) also find that Boötes II is best described by the most metal-poor fiducials.

To check these empirical results and to investigate a range of possible ages for Boötes II, we repeat the distance modulus fitting described in $\S 3.3$ using the theoretical isochrones of Dotter et al. (2008) in SDSS colors. We use 24 isochrones corresponding to the combinations with $[\mathrm{Fe} / \mathrm{H}]=-2.3,-1.5$, and -0.7 , ages of 5, 7, 10, and $13 \mathrm{Gyr}$, and $\alpha$-element abundances of $[\alpha / \mathrm{Fe}]=$ 0.0 and 0.2 . The best fitting of all 24 isochrones is that with $[\mathrm{Fe} / \mathrm{H}]=-2.3,13 \mathrm{Gyr}$, and $[\alpha / \mathrm{Fe}]=0.2$, with 98 stars having colors lying within 0.05 mag of the isochrone. For the same abundance values, the number of stars drops to 86,78 , and 72 for the 10,7 , and 5 Gyr populations, respectively. This quantitative comparison with the Dotter isochrones highlights the fact that the small color difference between the MSTO and RGB stars in Boötes II would not be consistent with a stellar population much younger than 13 Gyr.

\subsection{Structural Parameters}

The surface density profiles of globular clusters (GCs) and dwarf spheroidal galaxies (dSphs) are commonly parameterized by King (1966), Plummer (1911), and exponential profiles. To facilitate comparison with other observational studies, we fit all three profiles to the stellar distribution of Boötes II:

$$
\begin{gathered}
\Sigma_{\text {King }}(r)=\Sigma_{0, \mathrm{~K}}\left[\left(1+\frac{r^{2}}{r_{c}^{2}}\right)^{-1 / 2}-\left(1+\frac{r_{t}^{2}}{r_{c}^{2}}\right)^{-1 / 2}\right]^{2}, \\
\Sigma_{\text {Plummer }}(r)=\Sigma_{0, \mathrm{P}}\left(1+\frac{r^{2}}{r_{\mathrm{P}}^{2}}\right)^{-2},
\end{gathered}
$$




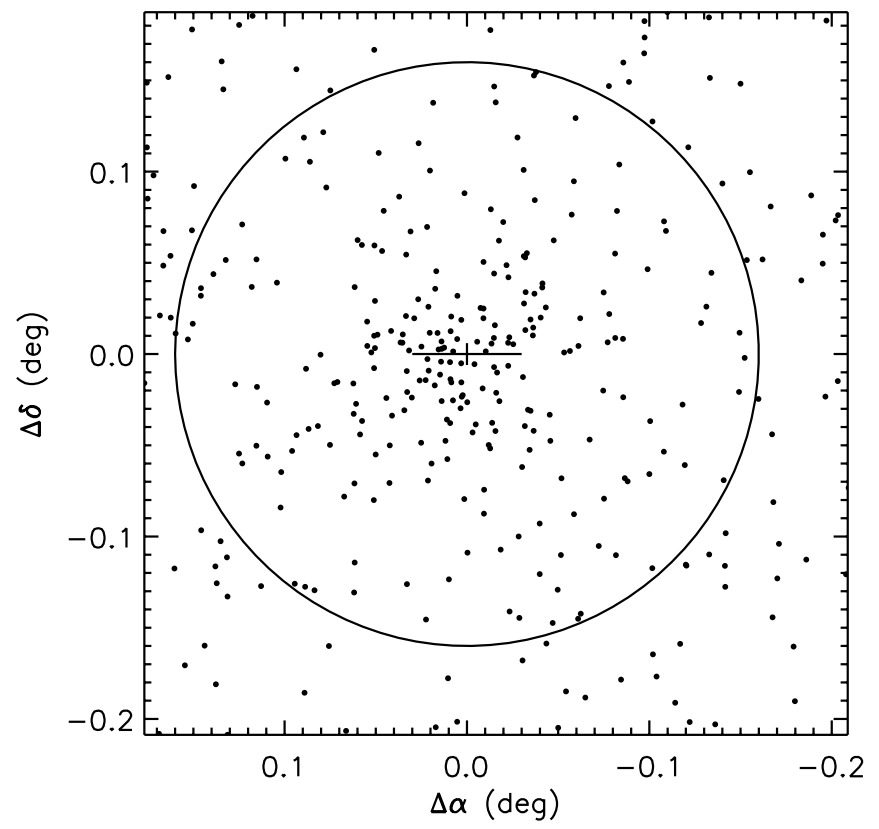

FIG. 3.- Positions of stars passing selection cuts. The calculated center of Boötes II is highlighted by the cross hair, which spans the R.A. and decl. uncertainties. The large circle shows the maximum radius of the surface density profile fit.

$$
\Sigma_{\exp }(r)=\Sigma_{0, e} \exp \left(-\frac{r}{\alpha}\right),
$$

where $r_{\mathrm{P}}$ and $\alpha$ are the scale lengths for the Plummer and exponential profiles, respectively, and $r_{c}$ and $r_{t}$ are the King core and tidal radii, respectively. For the Plummer profile, $r_{\mathrm{P}}$ equals the half-light radius $r_{h}$, while for the exponential profile $r_{h} \approx 1.668 \alpha$. The circled stars in the right panel of Figure 2 show the colormagnitude criteria we use to select probable Boötes II member stars for calculating its center and for investigating its structure. Figure 3 shows the spatial distribution of stars that pass these cuts and the location of their derived center. Figure 4 shows the surface density profile of Boötes II around this center, where the error bars were derived assuming Poisson statistics. Using a nonlinear least-squares method, we fit Plummer and exponential models, plus a constant field contamination, to this surface density profile. The surface density profile fits are only constrained to be physically possible systems (i.e., field contributions must be positive). In the case of the King profile, the tidal radius is present in a constant term; hence, there is a degeneracy between the field value and the tidal radius. To circumvent this, we first fix the King field value using the mean of the fitted Plummer and exponential field values.

All three fits yield consistent characteristic radii. Assuming a distance modulus of $m-M=18.1$, the Plummer and exponential profiles yield physical half-light radii of $r_{h \text {, Plummer }} \simeq 36 \pm 9 \mathrm{pc}$ and $r_{h \text {, exponential }} \simeq 33 \pm 10 \mathrm{pc}$, respectively. The King model fit yields a core radius of $r_{c} \simeq 25 \pm 9 \mathrm{pc}$ and a tidal radius of $r_{t} \simeq$ $155 \pm 35 \mathrm{pc}$. Although this tidal radius lies just outside the extent of the radial profile that we can measure, we find that the inner radial bins constrain the core radius and central density, while fixing the field value leaves only the tidal radius as a free parameter. If the outer radial bins are contaminated with Boo II stars, and are therefore higher than the true field value, the tidal radius could be much larger. As an example, if the Plummer and exponential models were to overestimate the true field value by $25 \%$, then the best-fit tidal radius would be $\sim 215 \mathrm{pc}$, while the

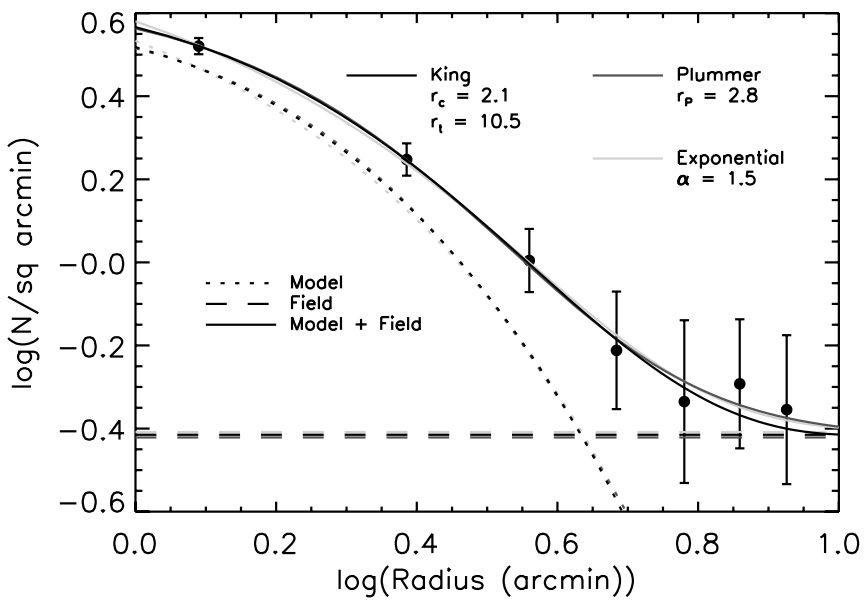

FIG. 4- - Fitted surface density profile of Boötes II consisting of a Plummer (blue lines), exponential (red lines), or King (black lines) profile, combined with a constant field contribution. [See the electronic edition of the Journal for a color version of this figure.]

core radius would remain relatively constant at $\sim 22 \mathrm{pc}$. The fitted King tidal radius of $r_{t} \simeq 155 \pm 35$ is a lower limit.

Figure 5 shows in gray scale the distribution of King core and tidal radii derived from the bootstrap of Boötes II stars. The measured core and tidal radii of Galactic globular clusters are overplotted. We have overplotted lines of constant concentration $\left(r_{\text {tidal }} / r_{\text {core }}\right)$ and the concentrations of known globular clusters calculated with the tidal and core radii in the catalog of Harris (1996). Only a handful of known globulars have $r_{h} \geq 10 \mathrm{pc}$, and the only one larger than $r_{h} \sim 20 \mathrm{pc}$ ( Pal 5) is known to be tidally disrupting. However, this figure shows several known globular clusters with a King concentration as low as that of Boötes II. The King concentrations of the Milky Way's dwarf spheroidals range from

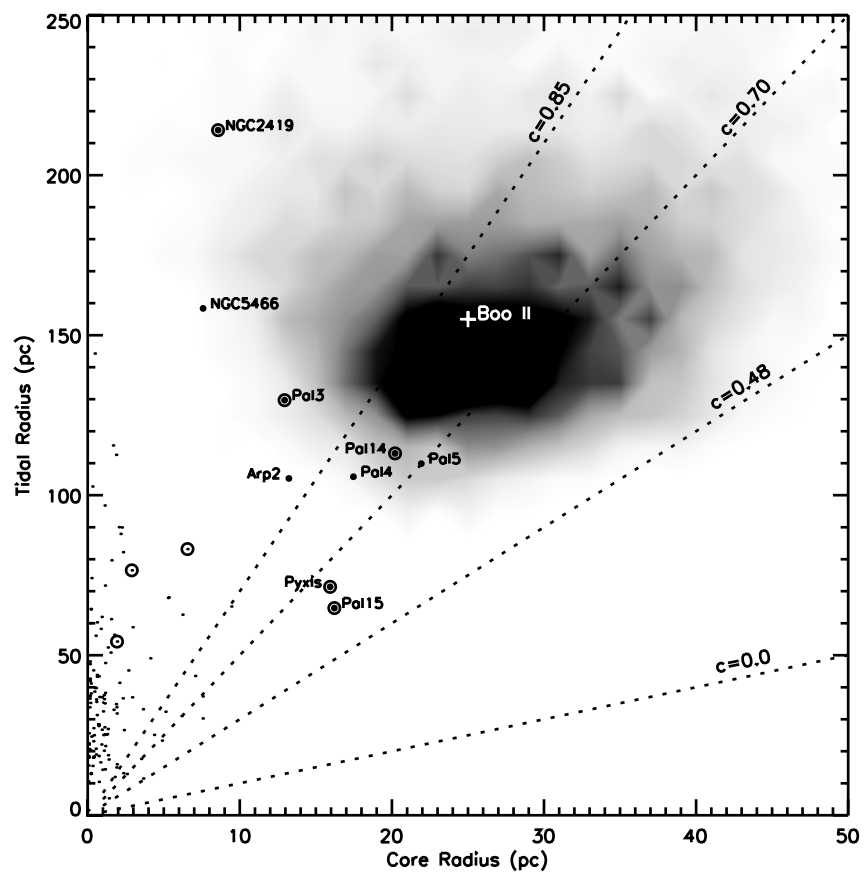

FIG. 5. - Two-dimensional histogram of bootstrap results for Boötes II's King core radius and tidal radius, with Galactic globular clusters (GGCs). GGCs with $r_{c}>10 \mathrm{pc}$ or $r_{t}>100 \mathrm{pc}$ are shown with larger points. Dashed lines show contours of constant concentration, defined as $c=\log \left(r_{t} / r_{c}\right)$. The open circles show the eight globulars with $35 \mathrm{kpc}<d<100 \mathrm{kpc}$, for which we calculate $M / L$ in $\S 4.3$. 


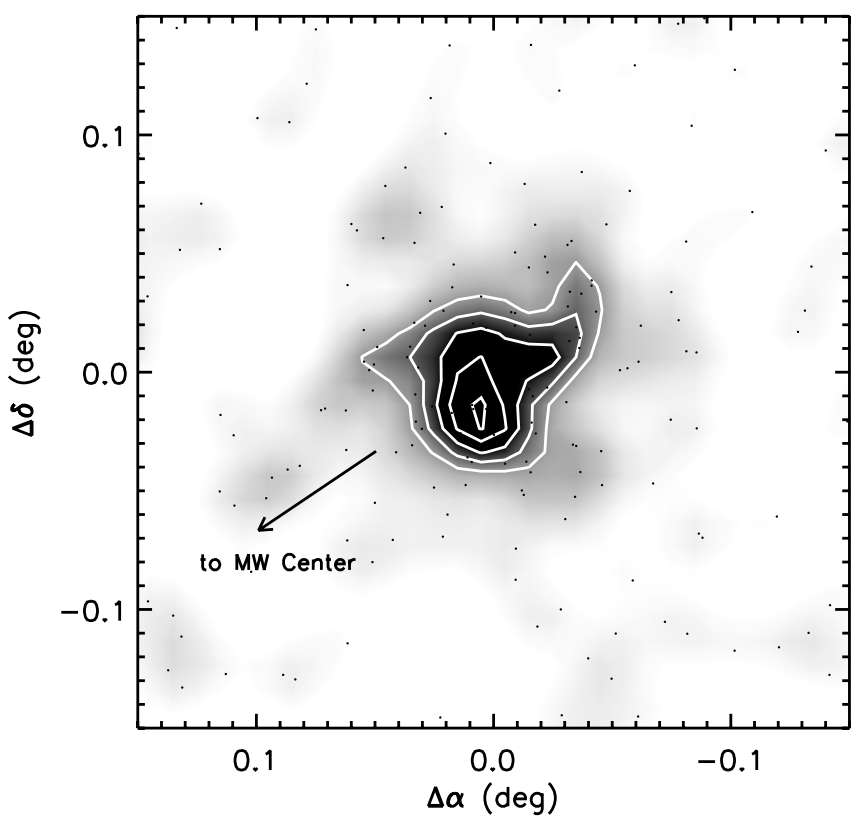

FIG. 6.-Smoothed contour plot of Boötes II showing an apparent distortion along the direction toward the Galactic center. The contours show the 3, 4, 5, and $6 \sigma$ levels above the mean.

0.48 to 1.12 (Mateo 1998), which is similar to the range of GCs in this figure. We do not overplot the King parameters of the classical Milky Way dwarf galaxies, because their relaxation times are approximately a Hubble time. Their King profile fits thus contain less physical meaning than those of objects with shorter relaxation times, such as globular clusters and Boötes II (see $\S 4$ for discussion).

\subsection{Luminosity}

To estimate the total luminosity of Boötes II, we integrate the model components of the surface density profile, corrected for incompleteness, to derive the number of Boötes II stars within the CMD selection cuts. Using this number to normalize the theoretical luminosity function gives estimates of $M_{r}=-2.6\left(M_{V}=\right.$ $-2.4)$ for the Plummer profile and $M_{r}=-2.4\left(M_{V}=-2.2\right)$ for the exponential profile (using $V-r=0.16$, adapted from Girardi et al. [2004] for a $13 \mathrm{Gyr},[\mathrm{Fe} / \mathrm{H}]=-2.27$ stellar population). We obtain these magnitudes after correcting for the missing flux from stars fainter than $r=23.5$ by integrating the theoretical luminosity function taken from Girardi et al. (2004) for a $[\mathrm{Fe} / \mathrm{H}]=-2.27$, 13 Gyr population. This method counts the number of stars without regard to their individual magnitudes, which could be strongly affected by the addition or subtraction of a single RGB star in systems of such low luminosity. Such a star's individual magnitude could be much brighter than $M_{V}=-2$, rendering a more traditional method of summing fluxes unreliable. Hence, a group of stellar systems in which each has an identical number of stars will have a spread in luminosity independent of any measurement uncertainty (see also Martin et al. 2008). Using simulated dwarf galaxies from Walsh et al. (2008), we find that for an object such as Boötes II as observed by MMT/Megacam, this spread has a standard deviation of $0.6 \mathrm{mag}$. We therefore combine this effect with the bootstrap uncertainty by summing in quadrature to derive the values presented in Table 1 .

\subsection{Morphology}

We look for evidence of tidal disturbance based on the morphology of Boötes II's isodensity contours. Binning the positions

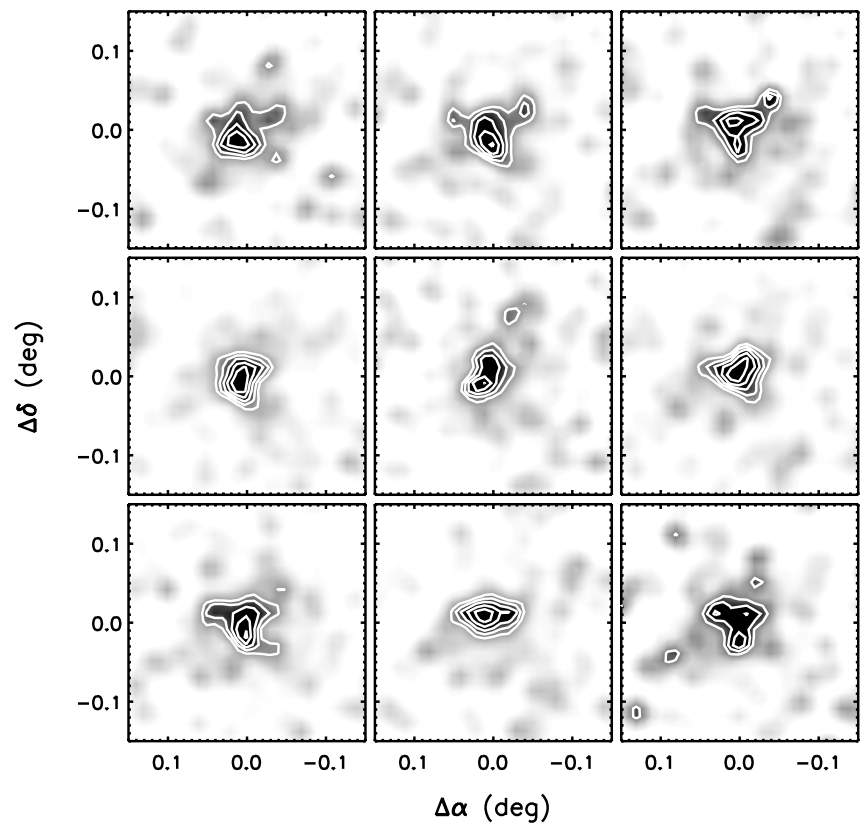

FIG. 7.- Smoothed contour plot of nine randomly selected resamples of Boötes II stars, showing varying morphology for each iteration. The contours show the $3,4,5,6$, and $7 \sigma$ levels above the mean.

of stars in Figure 3 into $0.01^{\circ} \times 0.01^{\circ}$ bins and spatially smoothing with a Gaussian of 1.5' FWHM scale length reveals an apparent distorted morphology to Boötes II, including substructure at the 3-5 $\sigma$ level and an elongation directed along the gradient of the Galactic potential, as shown in Figure 6. Although tidal tails trace an object's orbit, tidal debris is stripped from an object along the gradient of the gravitational potential such that tidal stars near an object are expected to lie along this gradient. The elongation of Boötes II could thus be a feature resulting from tidal interaction.

However, due to the meager number of stars in Boo II, any observed irregular morphology may be an effect of small-number statistics. To evaluate the significance of the morphology shown in Figure 6, we generate contour plots of bootstrap resamples of Boötes II stars. Figure 7 shows nine such randomly selected isodensity contours defined as in Figure 6. This figure shows that the irregular morphology and apparent distortion along the Galactic potential are not persistent features. While this does not rule out tidal disturbance to Boötes II, the varying morphologies of these resampled objects demonstrate that the shape of Boötes II does not necessarily reflect a true irregularity in its underlying spatial distribution.

In order to quantify any asymmetry in the morphology of Boötes II, we first derive the position angle $\theta$ and ellipticity $e$. We calculate these from the standard SExtractor definitions (Bertin \& Arnouts 1996) using the smoothed images, assuming that all pixels greater than $3 \sigma$ above the mean are part of Boötes II. We then count the number of stars on either side of the major and minor axes within $1.5 r_{h}$. If the positions of these stars are drawn from an axisymmetric distribution, then the numbers on either side of an axis should be within $(2\langle N\rangle)^{1 / 2}$ of each other. We define an asymmetry parameter $A$ :

$$
A=\frac{N_{1}-N_{2}}{\sqrt{2\langle N\rangle}},
$$

where $N_{1}$ and $N_{2}$ are the counts on either side of the axis, and $\langle N\rangle$ is their average. Hence, for each bootstrap iteration we have two 
values of $A$ : one for the major axis and one for the minor. Doing this for simulated Boo II-like objects drawn from a pure Plummer density profile yields as expected a distribution of $A$ with a mean of 0.0 and a standard deviation of $\sigma_{\text {sims }}=1.01$. The bootstrap yields two distributions with means of $A_{\text {minor }}=-0.23$ and $A_{\text {major }}=$ -0.18 , and standard deviations of $\sigma_{\text {minor }}=1.03$ and $\sigma_{\text {major }}=1.01$. The asymmetry of Boötes II is therefore not statistically significant.

These results imply that any apparent asymmetry in the distribution of Boötes II stars is well within that expected from a symmetric system, and is probably due to small-number statistics. Deeper and wider field imaging may provide the signal necessary to definitively measure whether Boötes II has extended, tidal structure.

\section{IS BOÖTES II DARK MATTER DOMINATED?}

As discussed in $\S 1$, one motivation for studying an individual ultra-low-luminosity object such as Boötes II in great detail is to determine whether it is a galaxy or a star cluster. Using our definition of a dwarf spheroidal galaxy, this boils down to determining whether or not the object is dark matter dominated (but see, e.g., Metz \& Kroupa 2007; Dabringhausen et al. 2008 for another interpretation of the Milky Way's dwarf companions). Understanding the properties of dark matter depends critically on identifying the smallest mass and length scales at which dark matter will cluster (e.g., Strigari et al. 2007b; Gilmore et al. 2007). The most direct way we have at present to investigate dark matter on the smallest scales is by using the least luminous galaxies as tracers of dark matter, and by pushing the envelope to find the smallest galaxies that can form.

In this section, we use a tidal argument to show why Boötes II may be dark matter dominated, and thus may be a dwarf galaxy, despite its very low luminosity $\left(L \sim 500 L_{\odot}\right)$. The lines of evidence presented in this section are circumstantial now, but are illustrative of arguments that could provide strong constraints on the correct classification for Boötes II if deeper, wider field imaging, kinematics, and/or spectroscopic abundance measurements are able to demonstrate whether Boötes II is self-bound or not.

\subsection{Separation in Size-Luminosity Space from Dwarf Galaxies and Globular Clusters}

In Figure 8, we compare the size and luminosity of Boötes II with that of other nearby, old, stellar populations. This figure shows Boötes II in the size-luminosity plane along with Milky Way globular clusters (Harris 1996; Koposov et al. 2007b GCs), M31 (McConnachie \& Irwin 2006; Zucker et al. 2004, 2007), and Milky Way dwarf satellites (Mateo 1998; Grebel et al. 2003; Belokurov et al. 2006, 2007; Willman et al. 2005a, 2005b; Zucker et al. 2006a, 2006b; Irwin et al. 2007). In this figure, which includes only old stellar populations, Boötes II, Wil 1, and Segue 1 occupy a somewhat unique place in size-luminosity space. The apparent lack of objects with half-light radii larger than those of Boötes II, Wil 1, and Segue 1, but smaller than the apparent minimum size of confirmed dwarf galaxies $(\sim 100 \mathrm{pc})$, is quite possibly an observational selection effect due to the faint central surface brightnesses of objects in that region of the size-luminosity plane.

Conversely, Boötes II, Wil 1, and Segue 1 have half-light radii 20-40 pc, an order of magnitude larger than the half-light size characteristic of similarly low luminosity globular clusters $(-4<$ $\left.M_{V}<-1\right)$. Low-luminosity Milky Way clusters in that size gap could have been detected by the SDSS searches of Koposov et al. (2007a) or Walsh et al. (2008). This gap between Boötes II, Willman 1, and Segue 1 and the known globular clusters is thus

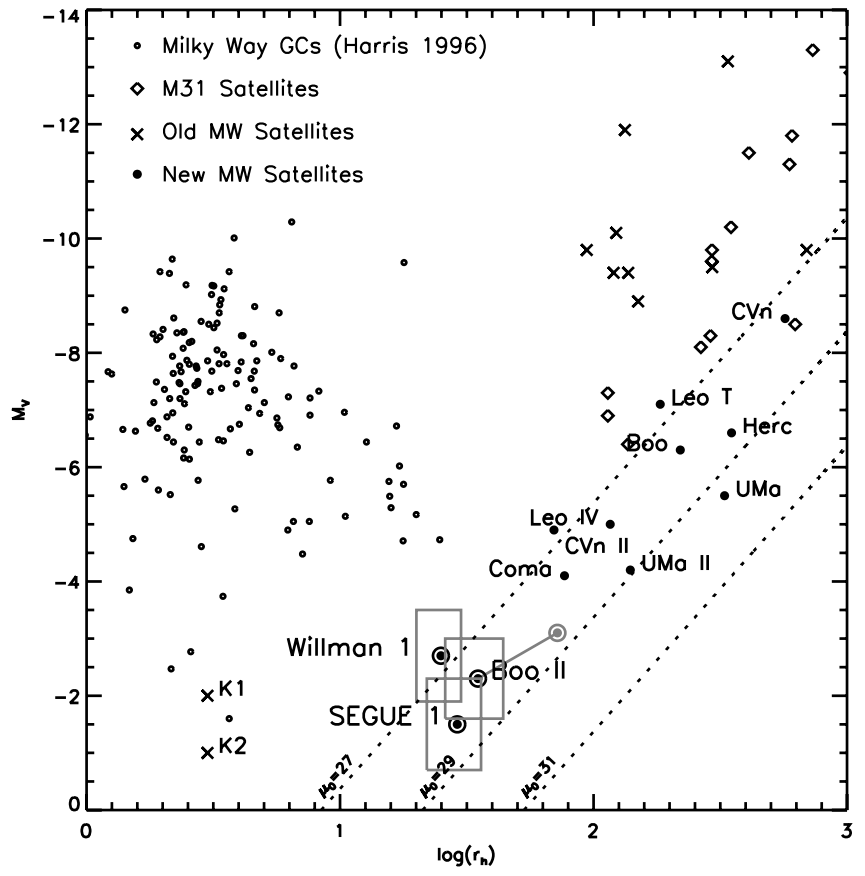

FIG. 8.- Size-luminosity plot of known Galactic satellites. The previous position of Boötes II is shown in gray along with the region bounded by the uncertainties in $r_{h}$ and $M_{V}$. Values for the new Milky Way satellites are taken from Martin et al. (2008). Globular cluster properties are from Harris (1996). Dotted lines show lines of constant surface brightness.

real and not a selection effect, which suggests that these objects are a population distinct from Milky Way GCs. However, if such diffuse old clusters do exist outside of the Milky Way, they would not yet have been discovered, owing to their low surface brightnesses. It thus remains possible that old star clusters with properties bridging the gap between Boötes II and known GCs do exist in abundance in other environments.

Based on the compilation of Dias et al. (2002), ${ }^{8}$ only two of the 1076 Milky Way open clusters in that catalog with both distance and diameter measurements have apparent radii larger than 20 pc. Young stellar associations in environments other than the Milky Way have been observed to have characteristic sizes of up to 100 pc (e.g., OB associations in the LMC observed by Gouliermis et al. 2003). Although the majority of these clusters appear to be unbound, some fraction of them could survive as self-bound entities for $10 \mathrm{Gyr}$ if subject only to very weak tidal forces. We will show in $\S \S 4.2$ and 4.3 that tidal effects limit the survivability of objects at Boötes II distances with star clusterlike mass-to-light ratios and sizes and luminosities similar to those of Boötes II, Willman 1, and Segue 1.

\subsection{Minimum Mass-to-Light Ratio to Be Bound}

We estimate a lower limit to the mass-to-light ratio required for Boötes II to be self-bound. We calculate the instantaneous tidal radius of Boötes II using

$$
r_{\text {tidal }} \propto\left(\frac{m_{\text {BooII }}}{3 M_{\mathrm{MW}}(d)}\right)^{1 / 3} d_{\text {BooII }},
$$

where $m_{\mathrm{BooII}}$ is the mass of Boötes II, $M_{\mathrm{MW}}(d)$ is the mass of the Milky Way within the distance to Boötes II, and $d_{\text {BooII }}$ is the

\footnotetext{
${ }^{8}$ Catalog obtained at http://www.astro.iag.usp.br/ wilton/.
} 
galactocentric distance to Boötes II. We calculate the Milky Way mass $M_{\mathrm{MW}}(d)$ assuming an isothermal sphere with a circular velocity of $V_{c}=220 \pm 40 \mathrm{~km} \mathrm{~s}^{-1}$ (Bellazzini 2004), using $d_{\text {BoolI }}=$ $42 \pm 2 \mathrm{kpc}$ and $M_{V}=-2.4 \pm 0.7 \mathrm{mag}$ for Boötes II. For $(M / L)_{V}$ values of $2,10,100$, and $1000 \mathrm{kpc}$, the corresponding tidal radii are thus $42_{-13}^{+19}, 72_{-21}^{+33}, 156_{-46}^{+70}$, and $336_{-99}^{+151} \mathrm{pc}$, respectively. A tidal radius of $72 \mathrm{pc}(M / L=10)$ is within our observed field and only $\sim 2$ times the half-light radius of Boötes II. The visible extent of Boötes II thus exceeds that expected for globular clusterlike mass-to-light ratios. If the Boötes II stars throughout our field of view are indeed bound to the object, then it requires a significant dark matter component. In $\S 3.7$, we showed that Boötes II lacks statistically significant tidal distortion, so there is presently no evidence that it is losing stars (however, see Muñoz et al. 2008). Deeper wide-field imaging and/or kinematic data will provide a clearer picture of the boundedness of this object.

\subsection{Mass-to-Light Ratio Inferred from the King Tidal Radius}

Using the same physical principle as in the previous section, we now calculate the mass-to-light ratio of Boötes II assuming that the King tidal radius derived in $\S 3.5$ is the true tidal radius of Boötes II, owing to the tidal field of the Milky Way. The King model (King 1966) is physically motivated and expected for a relaxed, single mass component, spherical system in equilibrium that is tidally limited by the gravitational field of the Milky Way. For velocity dispersions greater than $0.1 \mathrm{~km} \mathrm{~s}^{-1}$, the relaxation time of Boötes II is less than a Hubble time (unlike the classical Milky Way dSphs). Based on a Dotter et al. (2008) stellar luminosity function for a $13 \mathrm{Gyr},[\mathrm{Fe} / \mathrm{H}]=-2$ system normalized to have $M_{V}=-2.4 \mathrm{mag}$, Boötes II has 3700 stars more massive than $0.1 M_{\odot}$. Its relaxation time, $t_{\text {relax }} \sim N / 8 \ln (N) * t_{\text {cross }}$ (Binney $\&$ Tremaine 1987) at its Plummer half-light radius of $36 \mathrm{pc}$ is thus $\sim 1.1$ Gyr $\sigma_{v}^{-1}$, where $\sigma$ is its one-dimensional velocity dispersion. Although the presence of a significant dark component of matter would complicate the expected relaxation time of Boötes II, we are testing the hypothesis that it does not contain dark matter.

If we take the King tidal radius to be the instantaneous tidal radius of Boötes II, we can infer a mass $m_{\text {BooII }}$, and therefore a mass-to-light ratio. We use equation (7) as described in $\S 4.2$, but set $r_{\text {tidal }}=r_{\text {tidal,King }}=155 \pm 35 \mathrm{pc}$, and solve for the mass of Boötes II. We infer a $V$-band mass-to-light ratio of Boötes II of $98_{-84}^{+420}$. If this is representative of the true $M / L$ of Boötes II, its mass would be dominated by dark matter, classifying it as a galaxy. We note the substantial uncertainty on the $M / L$ derived in this way. Even if the input assumptions are robust, the dark matter signal is only significant at the $1 \sigma$ level. As mentioned in $\S 3.5$, the King tidal radius may be substantially larger if the outer surface density bins are contaminated with Boo II stars, thereby causing an overestimate of the field density. As in $\S 3.5$, if the field value is overestimated by $25 \%$, then the best-fit tidal radius would be $r_{t}=215 \mathrm{pc}$, and the inferred $M / L$ would be $263_{-216}^{+991}$.

For comparison, we use these same assumptions and the same technique to calculate the mass-to-light ratios of known Milky Way globular clusters in the distance range of $35 \mathrm{kpc} \leq R_{\mathrm{GC}} \leq 100 \mathrm{kpc}$. We chose this distance range because (1) these halo globulars reside in an environment and are on orbits that are the most comparable to Boötes II, and (2) Bellazzini (2004) states that the Milky Way mass profile is consistent with an isothermal sphere with a circular velocity of $V_{c}=220 \pm 40 \mathrm{~km} \mathrm{~s}^{-1}$ between 35 and $100 \mathrm{kpc}$. There are eight clusters in that range: Eridanus, Pal 2, NGC 2419, Pyxis, Pal 3, Pal 14, Pal 15, and NGC 7006. For all of these clusters, we use the distances and luminosities from the
Harris (1996) catalog and assign a distance uncertainty of $0.1 \mathrm{mag}$ in distance modulus. For Pal 2, NGC 2419, Pal 3, Pal 14, and NGC 7006, we use the core radii, concentrations, and uncertainties from McLaughlin \& van der Marel (2005). For the other three GCs, we use values from Harris (1996) and assign uncertainties of $10 \mathrm{pc}$ to their tidal radii. We find a median (mean) $(M / L)_{V}$ of $0.36_{-0.12}^{+0.98}\left(0.5_{-0.38}^{+1.85}\right)$ for these eight clusters. The single outlier with a calculated $M / L>1$ is Pal 14 with $M / L=2.4_{-1.9}^{+10.4}$. Although the true $M / L$ of most (all) of these eight halo globulars is within $1 \sigma(2 \sigma)$ of the calculated values, we find that this technique systematically underestimates the $M / L$ of these relaxed systems by up to an order of magnitude (three of the eight GCs have inferred $M / L<0.1$ ). This systematic underestimate is not surprising, because the tidal forces experienced by these halo globulars at this snapshot in time are smaller than the maximum tidal forces that they have experienced in their pasts. Using their present distance in this $M / L$ calculation will thus provide a lower limit on the masses required to yield the observed King tidal radii. Regardless, this calculation shows that Boötes II is an outlier from globular clusters with this metric of measurement.

\section{CONCLUSION}

In this paper, we use MMT/MegaCam imaging in $g$ and $r$ to present the first robust estimates of the fundamental properties of the ultra-low-luminosity Milky Way satellite Boötes II $(d \sim$ $42 \mathrm{kpc}$ ). This object is old, and its stellar population appears very similar to that of M92, showing that it is metal-poor $([\mathrm{Fe} / \mathrm{H}] \lesssim-2)$. With a total luminosity of only $\sim 500 L_{\odot}\left(M_{V} \sim-2.4 \pm 0.7 \mathrm{mag}\right)$ and a half-light size of $\sim 36 \pm 9$ pc (assuming a Plummer profile), Boötes II lies away from globular clusters and dwarf spheroidals, but near Willman 1 and Segue 1, in size-luminosity space. Although the morphology of Boötes II appears irregular and elongated along the direction of the Galactic potential, we showed that this distortion of its isodensity contours is not statistically significant in our data set.

The revised values we present for the distance, luminosity, and physical size of Boötes II differ from those originally estimated in the Walsh et al. (2007) discovery paper. The primary factor in these differences is the new distance estimate of $42 \mathrm{kpc}$ rather than $60 \mathrm{kpc}$. The SDSS discovery data was more than $2 \mathrm{mag}$ shallower than the data presented in this paper. VLT photometry of Boötes II will be presented in H. Jerjen et al. (in preparation), along with a more detailed discussion of its stellar population and of the possible association, or lack thereof, with the Sagittarius Stream.

Our bootstrap analysis demonstrated the impact of smallnumber statistics on the derived parameters for this ultrafaint class of objects, but showed that despite large uncertainties, Boötes II has a size that makes it distinct from Milky Way GCs, although its King concentration is similar to that of Milky Way dwarf galaxies, as well as to some diffuse GCs.

The gap between Boötes II, Willman 1, and Segue 1, and Milky Way globular clusters in size-luminosity space is not a selection effect, because existing surveys would have been sensitive to such objects. However, the apparent separation in half-light size at $100 \mathrm{pc}$ between dSphs and Boötes II, Willman 1, and Segue 1 could be a selection effect, making it more likely that these three objects are fundamentally connected with the dwarf galaxy population. We pointed out that old star clusters filling in that apparent gap may exist in environments other than the Milky Way, but would have escaped detection owing to their low surface brightnesses.

If Boötes II is a self-bound system in equilibrium, it could represent the continuation of the dwarf galaxy population into the 
extreme low-luminosity regime. We showed that it is reasonable to believe that Boötes II is a relaxed system, and used its King tidal radius to infer a lower limit $M / L$ of $\sim 98_{-84}^{+420}$. Dropping the assumption that the King tidal radius is physically meaningful for Boötes II, its spatial extent is larger than that naively expected for globular cluster-like mass-to-light ratios, if it is self-bound. However, we cannot rule out the possibility that it is a low- $M / L$ star cluster that is undergoing tidal disruption or disturbance, much like the Pal 5 cluster.

Regardless of whether or not Boötes II is dark matter dominated, it can provide a unique laboratory with which to investigate the low surface density limit of star formation and the tidal field of our Galaxy. The study in this paper also provides a partial road map for the future study of numerous similar objects that may be discovered in upcoming surveys for Galactic satellites to greater depth (e.g., PanSTARRS; Kaiser 2004) or in previously unsearched sky (e.g., Skymapper; Keller et al. 2007; Walsh et al. 2008). Ultimately, it will be necessary to determine the boundedness of the stars of the peculiar Boötes II object in order to definitively pin down its nature.
We thank the anonymous referee for improving this manuscript. We thank Bill Wyatt for maintaining a local copy of the SDSS data products at the Harvard-Smithsonian Center for Astrophysics. Jay Strader shared his transformations of the Clem et al. (2008) isochrones used in this paper and provided helpful conversations. We thank Brian McLeod for providing guidance and troubleshooting during our observing runs, writing software that our reduction relied on, and advising our MegaCam reduction. We thank Matt Ashby for creating the MegaCam reduction manual and for advising our reduction. We thank Maureen Conroy for troubleshooting during our observing runs, helping to create weight maps, and performing a preliminary coaddition of our Boötes II images. Mike Alegria, John McAfee, and Ale Milone provided observational support during our observing runs. Observations reported here were obtained at the MMT Observatory, a joint facility of the Smithsonian Institution and the University of Arizona. S. W. acknowledges partial financial support from the Australian Research Council Discovery Project grant DP0451426.

\section{Facilities: MMT (MegaCam)}

Adelman-McCarthy, J. K., et al. 2008, ApJS, 175, 297

Bellazzini, M. 2004, MNRAS, 347, 119

Belokurov, V., et al. 2006, ApJ, 647, L111 2007, ApJ, 654, 897

Bertin, E., \& Arnouts, S. 1996, A\&AS, 117, 393

Binney, J., \& Tremaine, S. 1987, Galactic Dynamics (Princeton: Princeton Univ. Press)

Cho, D.-H., Lee, S.-G., Jeon, Y.-B., \& Sim, K. J. 2005, AJ, 129, 1922

Clem, J., VandenBerg, D., \& Stetson, P. 2008, AJ, 135, 682

Dabringhausen, J., Hilker, M., \& Kroupa, P. 2008, MNRAS, 386, 864

Dias, W. S., Alessi, B. S., Moitinho, A., \& Lépine, J. R. D. 2002, A\&A, 389, 871

Dotter, A., Chaboyer, B., Jevremovic, D., Kostov, V., Baron, E., \& Ferguson, J. W. 2008, ApJS, 178, 89

Gilmore, G., Wilkinson, M. I., Wyse, R. F. G., Kleyna, J. T., Koch, A., Evans, N. W., \& Grebel, E. K. 2007, ApJ, 663, 948

Girardi, L., Grebel, E. K., Odenkirchen, M., \& Chiosi, C. 2004, A\&A, 422, 205

Gouliermis, D., Kontizas, M., Kontizas, E., \& Korakitis, R. 2003, A\&A, 405, 111

Grebel, E. K., Gallagher, J. S., III, \& Harbeck, D. 2003, AJ, 125, 1926

Grundahl, F., Stetson, P. B., \& Andersen, M. I. 2002, A\&A, 395, 481

Harris, J. 2007, ApJ, 658, 345

Harris, W. E. 1996, AJ, 112, 1487

Irwin, M. J., et al. 2007, ApJ, 656, L13

Kaiser, N. 2004, Proc. SPIE, 5489, 11

Keller, S., et al. 2007, Publ. Astron. Soc. Australia, 24, 1

King, I. R. 1966, AJ, 71, 64

Koposov, S., et al. 2007a, ApJ, submitted (arXiv:0706.2687)

2007b, ApJ, 669, 337

Kraft, R. P., \& Ivans, I. I. 2003, PASP, 115, 143

Martin, N. F., de Jong, J. T. A., \& Rix, H.-W. 2008, ApJ, 684, 1075

Martin, N. F., Ibata, R. A., Chapman, S. C., Irwin, M., \& Lewis, G. F. 2007, MNRAS, 380, 281

Mateo, M. L. 1998, ARA\&A, 36, 435

McConnachie, A. W., \& Irwin, M. J. 2006, MNRAS, 365, 1263

McLaughlin, D. E., \& van der Marel, R. P. 2005, ApJS, 161, 304

McLeod, B. A., Conroy, M., Gauron, T. M., Geary, J. C., \& Ordway, M. P. 2000, in Further Developments in Scientific Optical Imaging, ed. M. B. Denton (Cambridge: Royal Society of Chemistry), 11

\section{REFERENCES}

Metz, M., \& Kroupa, P. 2007, MNRAS, 376, 387

Muñoz, R. R., Majewski, S. R., \& Johnston, K. V. 2008, ApJ, 679, 346

Padmanabhan, N., et al. 2008, ApJ, 674, 1217

Paust, N. E. Q., Chaboyer, B., \& Sarajedini, A. 2007, AJ, 133, 2787

Plummer, H. C. 1911, MNRAS, 71, 460

Pritzl, B. J., Venn, K. A., \& Irwin, M. 2005, AJ, 130, 2140

Rider, C. J., Tucker, D. L., Smith, J. A., Stoughton, C., Allam, S. S., \& Neilsen, E. H., Jr. 2004, AJ, 127, 2210

Sakamoto, T., \& Hasegawa, T. 2006, ApJ, 653, L29

Schlegel, D. J., Finkbeiner, D. P., \& Davis, M. 1998, ApJ, 500, 525

Simon, J. D., \& Geha, M. 2007, ApJ, 670, 313

Sneden, C., Pilachowski, C. A., \& Kraft, R. P. 2000, AJ, 120, 1351

Stetson, P. B. 1994, PASP, 106, 250

Strigari, L. E., Bullock, J. S., Kaplinghat, M., Diemand, J., Kuhlen, M., \& Madau, P. 2007a, ApJ, 669, 676

Strigari, L. E., Kaplinghat, M., \& Bullock, J. S. 2007b, Phys. Rev. D, 75, 061303

Strigari, L. E., Koushiappas, S. M., Bullock, J. S., Kaplinghat, M., Simon, J. D., Geha, M., \& Willman, B. 2008, ApJ, 678, 614

Walker, M. G., Mateo, M., Olszewski, E. W., Gnedin, O. Y., Wang, X., Sen, B., \& Woodroofe, M. 2007, ApJ, 667, L53

Walsh, S. M., Jerjen, H., \& Willman, B. 2007, ApJ, 662, L83

Walsh, S. M., Willman, B., \& Jerjen, H. 2008, AJ, submitted (arXiv:0807.3345)

Willman, B., Dalcanton, J., Ivezić, Ž., Jackson, T., Lupton, R., Brinkmann, J.,

Hennessy, G., \& Hindsley, R. 2002, AJ, 123, 848

Willman, B., et al. 2005a, AJ, 129, 2692

2005b, ApJ, 626, L85

York, D. G., et al. 2000, AJ, 120, 1579

Zucker, D. B., et al. 2004, ApJ, 612, L121 2006a, ApJ, 650, L41 2006b, ApJ, 643, L103 2007, ApJ, 659, L21 